\title{
Fusion genes in acute myeloid leukemia: do acute myeloid leukemia diagnostics need to fuse with RNA-sequencing?
}

\section{Felicitas Thol}

\section{Department of Hematology, Hemostasis, Oncology, and Stem Cell Transplantation, Hannover Medical School, Hannover, Germany}

E-mail: FELICITASTHOL - thol.felicitas@mh-hannover.de

\section{doi:10.3324/haematol.2021.278983}

I $\mathrm{n}$ this issue of Haematologica, Kerbs and colleagues present a detailed analysis of fusion gene detection by RNAsequencing in acute myeloid leukemia (AML). ${ }^{1}$ Fusion genes, i.e., genes made by joining parts of two different genes, occur frequently in carcinogenesis. These fusion transcripts can occur within one chromosome as well as between two chromosomes (inter-chromosomal) and include translocations, deletions, insertions as well as inversions. Thousands of chromosomal aberrations and fusion genes have been identified as being recurrently present in cancer. ${ }^{2}$ Interestingly, in pediatric acute lymphocytic leukemia it was shown that many fusion genes are already present in utero and that a further transformation occurs in early childhood through secondary mutations. ${ }^{3}$ The discovery of $B C R-A B L 1$ in patients with chronic myeloid leukemia as well as gene fusions involving neurotrophic tyrosine receptor kinase (NTRK) ${ }^{4}$ and neuregulin-1 (NRG1) $)^{5}$ in solid cancers are examples of how the identification of fusion genes have not only revolutionized the diagnosis but also the therapy of a disease. It is estimated that fusion genes occur in $30 \%$ of patients with $\mathrm{AML}^{6}$ and it has been recognized that certain recurrent chromosomal and genetic aberrations delineate distinct types of AML. Along this line, the current World Health Organization (WHO) classification of AML describes seven types of recurrent genetic abnormalities in AML as well as the provisional entity "AML with BCR-ABL1". Furthermore, the WHO classification lists recurrent genetic aberrations that classify the disease as "AML with myelodysplasia-related changes". This underscores the relevance of identifying chromosomal aberrations and fusion genes in AML patients. The European LeukemiaNet recommends cytogenetics and screening for certain gene mutations for all AML patients at the time of diagnosis. ${ }^{8}$ Screening for gene rearrangements (e.g., with fluorescence in situ hybridization or reverse transcription polymerase chain reaction [RT-PCR]) should also be performed in some special circumstances (e.g., poor quality of chromosome morphology, need for rapid information). However, although cytogenetic analysis is still the gold standard, many small aberrations can be easily missed with this technique. RNA-sequencing is a next-generation sequencing technique that detects the presence and quantity of RNA and thus describes the transcriptome. Additionally, it gives information about alternative splicing, mutations, changes in gene expression, fusion genes, etc. We can expect additional information that we would not be able to obtain with routine cytogenetic analysis. Thus, RNA-sequencing can give us a deeper and dynamic picture of what is happening in cells.

Kerbs and colleagues applied RNA-sequencing to a cohort of 806 AML samples that also underwent conventional diagnostics with karyotyping and molecular diagnostics (RT-PCR and fluorescence in situ hybridization) (Figure 1). This allowed a direct comparison between results obtained with these different techniques. Special attention was paid to the detection of fusion genes. Here, $90 \%$ of fusion genes reported by conventional diagnostics were also detected by RNA-sequencing. Nevertheless, the fact that some fusion genes were not recog-

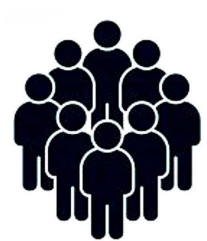

806 AML samples

Cohorts:

AMLCG

BEATAML

DKTK

FIMM
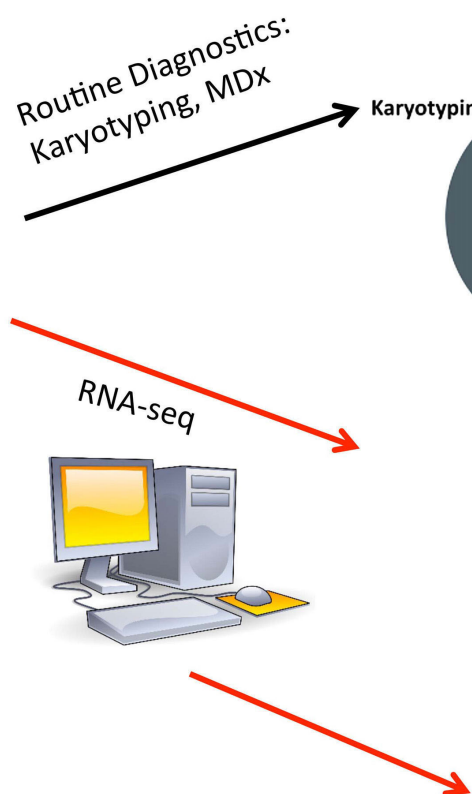

of a workflow

with integrated

filtering activity

$\downarrow$
True fusions

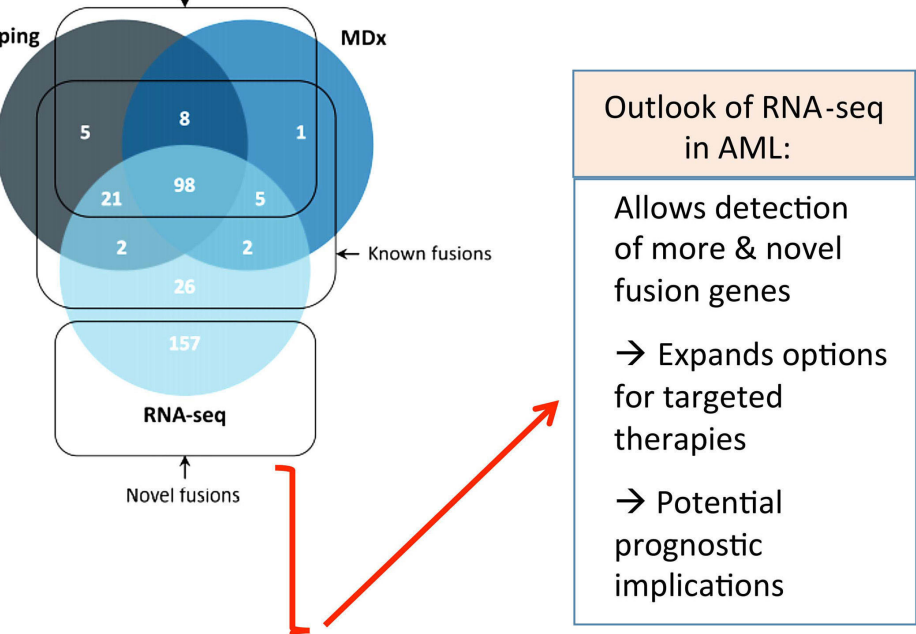

Figure 1. Design and outlook of an integrated analysis that includes routine diagnostics (karyotyping, molecular diagnostics) as well as RNA-sequencing in acute myeloid leukemia. In this case, 806 samples from four studies were investigated. RNA-seq: RNA-sequencing; MDx: molecular diagnostics; AML: acute myeloid leukemia. 
nized by RNA-sequencing indicates a weakness of this novel technique. Some fusion genes can be missed by RNA-sequencing if only low or uneven coverage is achieved. Thus, a high read depth of samples is essential for RNA-sequencing in order to detect all fusion genes. Furthermore, some affected genes might be more easily missed if they are not highly expressed (i.e., RNAsequencing is highly dependent on gene expression). However, RNA-sequencing allowed the authors to detect 26 cases with known recurrent fusion events that failed to be detected by routine diagnostics. Furthermore, Kerbs and colleagues also detected two novel recurrent fusion genes by RNA-sequencing. The fusion gene NRIP1MIR99AHG was found in $1.1 \%$ of patients, and the fusion gene LTN1-MX1 in $0.25 \%$ of patients. These are just examples that demonstrate that many more recurrent fusion genes will be detected if RNA-sequencing becomes employed more widely in AML. It is therefore very likely that we currently still underestimate the occurrence of fusion genes in AML with conventional diagnostics.

The encouraging results with RNA-sequencing also raise the questions of whether the technique should be applied to all AML patients at the time of diagnosis and whether it could replace conventional diagnostics. Currently, there are still a few obstacles to routine use of RNA-sequencing in all AML patients. Besides costs and labor-consuming aspects of this technique, analysis of results and data interpretation are very challenging. In particular, the high rate of false positive calls for fusion genes raises concerns and requires awareness. So far, interpretation of RNA-sequencing data has not been standardized and over 20 algorithms for fusion gene detection by RNA-sequencing have been described. ${ }^{9}$ However, standardization is a critical requirement for using this technique more widely and in routine clinical practice. In this sense, it is encouraging as well as important that Kerbs and colleagues have developed and report a workflow with integrated filtering strategies for the identification of robust fusion gene candidates by RNA-sequencing. Similar steps need to be undertaken in the future in order to implement RNA-sequencing in routine diagnostics. The wider use of RNA-sequencing in AML is likely to have positive impacts on diagnostic classification as well as prognostic stratification.

RNA-sequencing can also open the door to novel therapeutic strategies in individual AML patients. Gene fusions involving the NTRK gene constitute an encouraging example. NTRK fusions rarely occur in AML and are easily missed with routine diagnostics. ${ }^{10}$ Larotrectinib is a targeted therapy that has shown efficacy in a broad spectrum of NTRK fusion-positive cancers. Many fusion genes occurring through chromosomal translocations encode tyrosine kinases that are involved in signal transduction. The development of tyrosine kinase inhibitors represents an attrac- tive therapeutic strategy for patients with such fusions and RNA-sequencing will be essential for identifying these patients. It is therefore very likely that the increase in detection of recurrent gene fusions will also expand opportunities for targeted therapies in AML. RNA-sequencing may also have further implications for monitoring measurable residual disease in AML. Fusion genes are ideally suited for molecular measurable residual disease assessment as they can be detected by RT-PCR as well as next-generation sequencing very sensitively. ${ }^{11}$ Of note, RNA-sequencing is evolving as an upcoming standard in solid cancers as well.

In summary, the study by Kerbs and colleagues is an important step towards implementing RNA-sequencing in routine AML diagnostics. Further work is undoubtedly required (especially regarding standardizing data analysis), but the manuscript already gives an encouraging outlook of how RNA-sequencing can translate into clinical applications.

\section{Disclosures \\ No conflicts of interest to disclose.}

\section{References}

1. Kerbs P, Vosberg S, Krebs S, et al. Fusion gene detection by RNAsequencing complements diagnostics of acute myeloid leukemia and identifies recurring NRIP1-MIR99AHG rearrangements. Haematologica. 2021;107(1):100-111.

2. Mitelman DatabaseChromosome Aberrations and Gene Fusions in Cancer. https://mitelmandatabase.isb-cgcorg/. 2021. Last accessed June 30, 2021.

3. Wiemels JL, Cazzaniga G, Daniotti M, et al. Prenatal origin of acute lymphoblastic leukaemia in children. Lancet. 1999;354(9189):1499. 1503 .

4. Drilon A, Laetsch TW, Kummar S, et al. Efficacy of larotrectinib in TRK fusion-positive cancers in adults and children. $\mathrm{N}$ Engl J Med. 2018:378(8):731-739

5. Laskin J, Liu SV, Tolba K, et al. NRG1 fusion-driven tumors: biology, detection, and the therapeutic role of afatinib and other ErbB-targeting agents. Ann Oncol. 2020;31(12):1693-1703.

6. Grimwade D, Hills RK, Moorman AV, et al. Refinement of cytogenetic classification in acute myeloid leukemia: determination of prognostic significance of rare recurring chromosomal abnormalities among 5876 younger adult patients treated in the United Kingdom Medical Research Council trials. Blood. 2010;116(3):354-365.

7. Arber DA, Orazi A, Hasseriian R, et al. The 2016 revision to the World Health Organization classification of myeloid neoplasms and acute leukemia. Blood. 2016;127(20):2391-2405.

8. Dohner H, Estey E, Grimwade D, et al. Diagnosis and management of AML in adults: 2017 ELN recommendations from an international expert panel. Blood. 2017;129(4):424-447.

9. Haas BJ, Dobin A, Li B, Stransky N, Pochet N, Regev A. Accuracy assessment of fusion transcript detection via read-mapping and de novo fusion transcript assembly-based methods. Genome Biol. 2019:20(1):213

10. Joshi SK, Qian K, Bisson WH, et al. Discovery and characterization of targetable NTRK point mutations in hematologic neoplasms. Blood. 2020;135(24):2159-2170.

11. Thol F, Gabdoulline R, Liebich A, et al. Measurable residual disease monitoring by NGS before allogeneic hematopoietic cell transplantation in AML. Blood. 2018;132(16):1703-1713. 